\title{
44 Victimization of Sulfur Mustard in Qiqihar: Case Reports
}

\author{
Jinghai Sun, Wei Zheng* \\ The 208th Hospital, Changchun, China. \\ Email: *ZW0113@sina.com \\ Received September $10^{\text {th }}$, 2012; revised October $28^{\text {th }}$, 2012; accepted November $28^{\text {th }}, 2012$
}

\begin{abstract}
Background: Sulfur mustard (2,2'-bis-chloroethyl-sulfide; SM) has been a military threat since the World War I. SM is the major chemical warfare agent used by Japan during World War II. The clinical picture of poisoning, including cutaneous blisters, respiratory tract damage, ocular lesions and bone marrow depression is well known. In this report, we describe a civilian exposure in August 2003, in Qiqihar, Northeast China's Heilongjiang Province. Aim: To describe the clinical features and treatment of sulfur mustard toxicity. Results: In 44 victims of SM exposure, 32 had ocular symptoms; 43 showed significant skin blistering and pigmentation; 15 had pulmonary symptoms such as productive cough occurred; 18 have Central nervous symptoms and other effects. Conclusion: The 44 victims were all injured, when five barrels of mustard gas were dug up at a construction site in Qiqihar. The gas leak killed one and injured 43 others, one of the worst accidents involving chemical weapons abandoned by Japanese invading troops in China after WWII.
\end{abstract}

Keywords: Chemical Warfare Agents; Sulfur Mustard; Qiqihar; Toxicology

\section{Introduction}

Sulfur mustard is one of the most important chemical warfare agents developed and extensively used during World War I (WWI). It is synthesized already in 1822 by Despretz and 1860 by Niemann and Guthrie [1]. Its synonyms are sulfur mustard (typical odor), yperite (Ypres, place of the first military use), and yellow cross (German shells with vesicants were labeled with a yellow cross).

Pure SM is colorless and odorless. Due to impurities during the manufacturing process, technical grade sulfur mustard has a dark oily appearance and a typical odor of mustard or garlic. It is barely soluble in water.

Blistering after skin contact is one of the most prominent features of SM. Except cutaneous blisters, SM also cause respiratory tract damage, ocular lesions and bone marrow depression [2]. Most of the clinical symptoms can be explained by the strong alkylating characters, which attacks nearly all cell constituents. Each 2-chloroethyl side chain of sulfur mustard can react with proteins, RNA and DNA. DNA damage is believed to be the most important lesion [3]. Sulfur mustard is able to form nonfunctional adducts as well as interstrand crosslinks [4].

During World War II, large quantities of chemical weapons were abandoned or buried by Japanese troops in China. After 1949, the toxic gases were discovered in

*Corresponding author. central, eastern and southern China. Qiqihar, occupied by Japanese troops for 14 years between 1931 and 1945, was one of the chemical weapon exploitation departments of the Japanese forces.

\section{Patients and Background Information}

On August 4, 2003, one person was killed and 43 others wounded when several barrels of mustard gas abandoned by Japanese forces in 1945 leaked at a construction site in urban Qiqihar. When surveying nearby areas, police found many similar abandoned shells.

At 21:50 pm, on August 4, 2003, 3 victims were sent to the 203th hospital with symptom of cutaneous blisters and ocular lesions. 2 victims were hospitalized at 6:00 am, on August 5, and 39 others in the following 10 days. After treatment, the last patients recovered on November 15, 2003.

The 44 victims presented here were treated in The 203th Hospital of PLA in Qiqihar (39 males and 5 females, the median age was 29 ranging from 8 to 55). All the patients were recovered except 1 patient died as a result of a failure of multiple organs.

\section{Results}

\subsection{Clinical Signs and Symptoms}

Eyes: 32 (73\%) patients described conjunctivitis, lacri- 
mation, blurred vision, pain and photophobia. 30 (68\%) patients presented severe conjunctivitis. 7 (16\%) erosion of the cornea were the most severe ocular complication.

Skin: 43 (98\%) patients presented blistering characterizes significant dermal injury from sulfur mustard. The affected areas include body (10/43, 23\%), limbs (31/43, $72 \%)$, scrotum $(20 / 43,47 \%)$, buttocks $(10 / 43,23 \%)$, vulva $(5 / 43,12 \%)$, others $(2 / 43,5 \%)$. This shows that SM first invaded in direct contact and susceptible site. Exposed parts of the skin and tender part such as scrotum, vulva wet is easily injured.

Respiratory system: 15 (34\%) patients described respiratory tract damage. 9 of them presented a sore throat. 6 of them presented significant lung symptoms developed, such as coughing and sputum.

Other symptoms: 18 (41\%) patients described other symptoms include dizziness (10/18, 56\%), headache (9/18, 50\%), nausea (17/18, 94\%), followed by vomiting (10/18, 56\%), loss of appetite (3/18, 17\%).

\subsection{Treatment}

Eyes: eyes were washed with saline and antibiotic drops were used for 5 consecutive days. At the same time, antibiotic ointment was used to prevent infection and symblepharons. Atropine was applied to patients with keratitis and iridocyclitis to prevent synechia.

Skin: patients were washed daily with water (or 1.25\% sodium bicarbonate). Sulfadiazine-silver creme was applied to prevent infection. Recombinant human epidermal growth factor was used to promote wound healing.

Respiratory tract: codeine was given to relieve persistent cough. Inhalation of moist air including Gentamicin and Dexamethasone were used.

Other symptoms: glutamine, Recombinant human epidermal growth factor and Zadaxin were used to corresponding symptoms.

\section{Discussion}

Sulfur mustard (SM), also known as mustard gas, is an alkylating compound used as a chemical weapon in World War I. It is an lipophilic liquid with poor aqueous solubility yet is highly soluble in organic solvents $[5,6]$. Its color ranges from light yellow to dark brown, and with smells of garlic, horseradish, or mustard.

Once absorbed, sulfur mustard forms a reactive cyclic structure, the episulfonium ion, this ion is capable of reacting with DNA, RNA, proteins, carbohydrates, and lipids [7]. Despite its ability to attack multiple cell constituents, by cross-linking with DNA it induces the lethal lesion ultimately resulting in blister formation $[8,9]$.

Sulfur mustard exposure primarily affects the skin, respiratory tract, and eyes, though as an alkylating agent it can be toxic to rapidly proliferating cells, such as bone marrow and lymphoid tissue. The degree and onset of injury depends on multiple factors such as dose, mode of exposure, environmental conditions, and use of protective equipment.

Ocular injuries: the conjunctiva was the main site of injury, followed by the eyelid, and then the cornea and the iris. The length of the incubation period varied with the degree of injury and contact time and mode. Thorough washout and medicinal intervention should be instituted as early as possible.

Dermal damage: it is characterized by a latent period of 2 to 6 hours, followed by burning sensation, erythema, itching and swelling. After 18 to 24 hours, small vesicles may appear in erythema area and gradual integration into large blisters. The blisters rarely cause pain. But patients usually feel swollen, oppression.

Respiratory tract injury: the incubation period is 6 to 12 hours or longer. Its clinical manifestations are similar to symptoms of bad cold or bronchitis. Necrosis of bronchial mucosa leads to pseudo membranous which cause atelectasis and serious ventilation disorders. Patients with severe injury may describe chest pain, increased cough, fever, breathing difficulties and other clinical manifestations.

Other symptoms: other patients showed weakness, insomnia, headache, loss of appetite, nausea and vomiting.

4 - 6 weeks' recovery is necessary for the patients. However, after discharge, chronic health effects of SM exposure which are discussed later in this issue [10] were and are likely to affect them for the remainder of their lives.

\section{REFERENCES}

[1] F. Guthrie, "Ueber Einige derivate der Kohlenwasserstoffe CnHn,” European Journal of Organic Chemistry, Vol. 113, No. 3, 1860, pp. 266-288.

[2] B. Papirmeister, A. J. Feister, S. I. Robinson and R. D. Ford, "Medical Defense against Mustard Gas: Toxic Mechanisms and Pharmacological Implications," CRC Press, Boca Raton, 1991.

[3] I. J. Lodhi, J. F. Sweeney, R. E. Clift and D. B. Hinshaw, "Nuclear Dependence of Sulfur Mustard-Mediated Cell Death,” Toxicology and Applied Pharmacology, Vol. 170, No. 1, 2001, pp. 69-77. doi:10.1006/taap.2000.9083

[4] P. D. Lawley, J. H. Lethbridge, P. A. Edwards and K. V. Shooter, "Inactivation of Bacteriophage t7 by Mono- and Difunctional Sulfur Mustards in Relation to Cross-Linking and Depurination of Bacteriophage DNA,” Journal of Molecular Biology, Vol. 39, No.1, 1969, pp. 181-198. doi:10.1016/0022-2836(69)90341-6

[5] M. Balali-Mood and M. Hefazi, "The Pharmacology, Toxicology, and Medical Treatment of Sulfur Mustard Poisoning," Fundamental \& Clinical Pharmacology, Vol. 19, No. 3, 2005, pp. 297-315.

doi:10.1111/j.1472-8206.2005.00325.x 
[6] J. C. Dacre and M. Goldman, "Toxicology and Pharmacology of the Chemical Warfare Agent Sulfur Mustard," Pharmacological Reviews, Vol. 48, No. 2, 1996, pp. 290326.

[7] M. P. Shakarjian, D. E. Heck, J. P. Gray, et al., "Mechanisms Mediating the Vesicant Actions of Sulfur Mustard after Cutaneous Exposure,” Toxicological Sciences, Vol. 114, No. 1, 2010, pp. 5-19. doi:10.1093/toxsci/kfp253

[8] D. S. Rosenthal, C. M. Simbulan-Rosenthal and S. Iyer, "Sulfur Mustard Induces Markers of Terminal Differentiation and Apoptosis in Keratinocytes via a Ca-Calmodu- lin and Caspase-Dependent pathway,” Journal of Investigative Dermatology, Vol. 111, No. 1, 1998, pp. 64-71. doi:10.1046/j.1523-1747.1998.00250.x

[9] W. J. Smith, K. M. Sanders, S. E. Ruddle, et al., “Cytometric Analysis of DNA Changes Induced by Sulfur Mustard," Cutaneous and Ocular Toxicology, Vol. 12, No. 4, 1993, pp. 337-347. doi:10.3109/15569529309050150

[10] M. Rowell, K. Kehe, F. Balszuweit and H. Thiermann, "The Chronic Effects of Sulfur Mustard Exposure," Toxicology, Vol. 263, No. 1, 2009, pp. 9-11. doi:10.1016/j.tox.2009.05.015 